\title{
Western dietary pattern is related to premenstrual syndrome: a case-control study
}

\author{
Negin Farasati ${ }^{1}$, Fereydoun Siassi ${ }^{1}$, Fariba Koohdani ${ }^{2 *}$, Mostafa Qorbani ${ }^{3,4}$, Karolin Abashzadeh $^{1}$ and \\ Gity Sotoudeh ${ }^{1 *}$ \\ ${ }^{1}$ Department of Community Nutrition, School of Nutritional Sciences and Dietetics, Tehran University of Medical Sciences, \\ Tehran 14155/6117, Iran \\ ${ }^{2}$ Department of Cellular, Molecular Nutrition, School of Nutritional Sciences and Dietetics, Tehran University of Medical \\ Sciences, Tehran 14155/6117, Iran \\ ${ }^{3}$ Department of Community Medicine, School of Public Health, Alborz University of Medical Sciences, Karaj, Iran \\ ${ }^{4}$ Non-Communicable Diseases Research Center, Endocrinology and Metabolism Population Sciences Institute, Tehran \\ University of Medical Sciences, Tehran 32564765, Iran
}

(Submitted 12 May 2015 - Final revision received 28 August 2015 - Accepted 2 September 2015 - First published online 13 October 2015)

\begin{abstract}
Although premenstrual syndrome (PMS) affects a large number of women of reproductive age, the aetiology of this disorder has not yet been fully elucidated. The relationship between food intake and PMS morbidity has been investigated in several studies, but dietary patterns of PMS patients have not been taken into consideration up to now. We examined dietary patterns of 320 nurses with ( $n$ 160) and without ( $n$ 160) PMS in a case-control study. Food intakes over the past year were determined using semi-quantitative FFQ. Factor analysis was used to identify the main dietary patterns, and logistic regression was used to model the relationship between dietary patterns and PMS morbidity. Three dietary patterns were identified in the analysis: healthy, Western and traditional. After adjustment for age, BMI, menstrual cycles, physical activity and energy intake, participants in the second (OR 2.53; $95 \%$ CI 1.18, 5.43) and third (OR 4.39; $95 \%$ CI 1.97, 9.81) quintiles of the Western dietary pattern were more likely to experience PMS compared with those in the first quintile. The study findings therefore reveal that Western dietary pattern might be associated with PMS morbidity. However, this result should be interpreted with caution as there was no evidence of a dose-response relationship. Future studies are needed to confirm our findings in other populations.
\end{abstract}

Key words: Premenstrual syndrome: Food intake: Dietary patterns: Western dietary pattern

Premenstrual syndrome (PMS) is the most prevalent disorder in reproductive age women. According to the American College of Obstetricians and Gynecologists' diagnostic criteria, PMS symptoms are classified as affective and somatic symptoms such as anger, anxiety, social withdrawal, depression, irritability, headache and swelling. Up to $85 \%$ of menstruating women have one or more premenstrual symptoms, and $2-10 \%$ of this population are severely affected. Although patients experience negative effects on quality of life, the aetiology of PMS is not entirely clear ${ }^{(1,2)}$.

Several studies report that women with PMS may be so physiologically hypersensitive to the normal cycling levels of oestrogen and progesterone that they experience more symptoms ${ }^{(3)}$. Although twin studies suggest a heritable component, laboratory results from women with PMS demonstrate that an excess or deficiency of certain nutrients may result in hormonal and neurotransmitter imbalance ${ }^{(4,5)}$. A study that compared premenopausal women following a Western diet containing high fat and low fibre with vegetarians revealed that a low-fat vegetarian diet decreased plasma oestrogen levels and the duration of premenstrual symptoms ${ }^{(6,7)}$. Changing dietary guidelines towards consuming more complex carbohydrates and vegetable proteins, less simple carbohydrates, animal proteins and SFA and increasing fibre intake for 3-6 months resulted in raised serum progesterone levels, whereas serum oestradiol $17-\beta$ fell in the middle of the luteal phase ${ }^{(8)}$. Results from food-intake studies show that excess consumption of sweet-tasting food items, fast food, deep-fried meals, coffee and alcohol and low intakes of vegetables and fruits are significantly related to PMS incidence ${ }^{(9,10)}$.

Nursing is one of the most stressful jobs with lots of responsibilities and mental pressure. Research on work stress revealed that PMS symptoms in women with high responsibilities can be more severe than that of in others ${ }^{(11)}$. A study among Korean nurses revealed that $78.3 \%$ were suffering from PMS symptoms ${ }^{(12)}$. No study has yet evaluated the relationship

Abbreviations: PMS, premenstrual syndrome; PSST, premenstrual symptoms screening tool.

* Corresponding authors: G. Sotoudeh, fax +98 21 88974462, email gsotodeh@tums.ac.ir; F. Siassi, fax +98 21 88974462, email siassif@tums.ac.ir 
between dietary patterns and PMS. As nurses are at risk of more PMS morbidity, the aim of our study was to investigate the relationship between dietary patterns and PMS in nurses.

\section{Methods}

\section{Participants}

This case-control study was performed from February to October 2014 in nurses aged 20-45 years. Eight hospitals were randomly selected from seventeen hospitals affiliated to Tehran University of Medical Sciences, Iran. A list of all female nurses at these eight hospitals was prepared. Nurses were asked to fill in a premenstrual symptoms screening tool (PSST) questionnaire consecutively until 160 nurses with PMS and 160 nurses without PMS were selected for the study.

Exclusion criteria were as follows: presence of any diagnosed diseases, including diabetes, liver and kidney dysfunction, CVD, cancer, and polycystic ovary syndrome or premature menopause, $B M I>39.9 \mathrm{~kg} / \mathrm{m}^{2}$ or taking of contraceptive pills, anti-depressant and other psychotropic drugs, or thyroid and other endocrine drugs, or taking multivitamin and mineral supplements at least three times a week; women who were on a diet or those who smoked were also excluded. Furthermore, pregnant and lactating individuals were not included in the study. Ethical approval was obtained from the Medical Ethics Committee of Tehran University of Medical Sciences. A written informed consent was obtained from each participant after an explanation of the objectives and procedures of the study. We used four different instruments including: PSST questionnaire, socio-demographic questionnaire, semi-quantitative FFQ and International Physical Activity Questionnaire (IPAQ).

Participants completed the PSST questionnaire, and according to PMS criteria they were divided into two groups: PMS group ( $n$ 160) and healthy group $(n 160)$ as controls.

\section{Premenstrual symptoms}

The PSST questionnaire consists of nineteen questions in two parts. The first fourteen questions are about the intensity of somatic and affective symptoms. The five remaining questions evaluate the effect of symptoms on social connections. Each item is rated on a scale of $0-3$ points (not at all, mild, moderate and severe). According to the manual, the PMS group should have at least four moderate or severe symptoms from the first part (the first fourteen questions) with at least one being from the first four symptoms. Moreover, they must have one moderate or severe check mark from part two (the last five questions). The PSST is a simple user-friendly screening tool based on the fourth edition of Diagnostic and Statistical Manual of Mental Disorders' criteria ${ }^{(13)}$. The validity of the Iranian version of the PSST questionnaire has been demonstrated previously ${ }^{(14)}$.

\section{Socio-demographic characteristics}

The socio-demographic questionnaire consisted of general information such as age, marital status, educational level, position group, position ward, work shift, menstrual cycle status and bleeding period. In this study, regular menstrual cycle was defined as a normal period lasting $3-7 \mathrm{~d}$ in a $21-35 \mathrm{~d}^{\mathrm{cycle}}{ }^{(15)}$.

\section{Anthropometric measurements}

Participants were required to stand on digital scales (Omron BF508 0.1-150 kg; OMRON HEALTHCARE Co., Ltd) to measure their weight to the nearest $100 \mathrm{~g}$ while wearing light clothes without shoes. Standing height was measured without shoes to the nearest $0.5 \mathrm{~cm}$ using a tape measure while shoulders were in a relaxed position. To calculate the BMI, we divided the weight (kg) by square of height $\left(\mathrm{m}^{2}\right)$. Waist circumference was measured at the mid-point between the last rib and the iliac crest to the nearest $0.5 \mathrm{~cm}$ at the end of normal expiration using a non-elastic tape and without exerting any pressure on the body.

\section{Assessment of food intakes}

Intakes of macro- and micronutrients during the previous year were measured using a semi-quantitative FFQ. Participants were asked how frequently they consumed a specific portion size of 147 food items. We then noted down and multiplied the portion size and frequency of eating each item in order to obtain the total intake for the previous year. To save the nurses' time, we divided the FFQ into three parts, and each time we completed forty-nine items. The validity and reliability of the FFQ has been evaluated previously ${ }^{(16)}$.

\section{Physical activity measurement}

The short form of IPAQ was used to assess physical activity of participants during the previous $7 \mathrm{~d}^{(17)}$. The questionnaire includes seven items evaluating vigorous and moderate intensity of activities and walking for at least $10 \mathrm{~min} / \mathrm{d}$ during the previous week. To compute activities, we multiplied the duration and number of activity days by the metabolic equivalent task (MET) value of the activity and summed all activities to gain an overall estimate of total physical activity.

\section{Statistical analysis}

The Statistical Package for Social Sciences, version 15 (SPSS Inc.), was used for analysis. To compare the socio-demographic characteristics between the two groups, first we assessed the normality of variables via the Shapiro-Wilk's test. Variables with $P>0.05$ were considered as normal. We employed independent $t$ tests and Mann-Whitney's $U$ tests for continuous variables and $\chi^{2}$ tests for categorical variables. To ascertain the dietary patterns, factor analysis was performed. According to the similarity of food items, twenty-five food groups were created (Table 1). To explain sampling adequacy and inter-correlation of variables, Kaiser-Meyer-Olkin value and Bartlett's test of sphericity were marked. The principal components method, scree plot and eigenvalues over 1.4 were used to select the number of factors and extract the main dietary patterns. Varimax rotation was carried out to create a simple and definitive component matrix. Factor loading weighted the consumption of each food group to produce the factor score for each pattern, 
Table 1. Food groupings used in factor analysis

\begin{tabular}{ll}
\hline Groups & Subgroups \\
\hline Red and visceral meats and fast foods & Beef, lamb, ground meat, liver, heart, kidney, hamburger, sausage, kielbasa, pizza \\
Fish & Fish, canned fish \\
Poultry & Chicken \\
Eggs & Eggs \\
Low-fat dairy products & Milk, yogurt, cheese, dough \\
High-fat dairy products & Milk, cocoa milk, yogurt, cheese, ice cream, dried whey, cream \\
Tea and coffee & Tea, green tea, coffee \\
Cabbage & Cabbage \\
Green leafy vegetables & Spinach, lettuce \\
Orange and red vegetables & Carrot, tomato, tomato sauce \\
Other vegetables & Eggplant, celery, cucumber, pea, string bean, garlic, onion, pepper, mushroom, turnip, local vegetables, etc. \\
Cooked potato & Cooked potato \\
Fried potato & Fried potato \\
Whole grains & Maize, barley, wholegrain bread \\
Refined grains & White breads, baguettes, rice, spaghetti, vermicelli \\
Salty snacks & Chips, cheese puffs, crackers \\
Olive & Olive oil, olive seed \\
Sweets and desserts & Biscuits, cakes, cookies, halva \\
Pickles & Pickles \\
Hydrogenated oil & Butter, margarine, solid oil, animal oil \\
Vegetable oil and mayonnaise & Vegetable oils, mayonnaise \\
Fruit and juice & Dried fruit, fruit juices, canned fruit, cantaloupe, melon, watermelon, pear, apricot, cherry, apple, peach, fig, \\
Sugar and soft drinks & grape, kiwi, orange, plum, banana, strawberry, mulberry, persimmon, etc. \\
Nuts and legumes & Soft drinks, sugar, chocolate, gaz, sohan, honey, jam, candy \\
Spices & Almonds, pistachios, hazelnuts, walnuts, seeds, beans, lentils, dried peas, soya \\
\hline
\end{tabular}

and each subject got a particular factor score for each of the three patterns. Then, based on quintile, factor scores were classified into five groups. Logistic binary regression was used to assess the association of quintile of dietary pattern scores with incidence of PMS among groups. Two models were formed: model 1 was crude, and model 2 was controlled for age, menstrual cycle status, BMI, energy intake and physical activity score.

\section{Results}

Characteristics of cases and controls are defined in Table 2. There were no significant differences in quantitative variables between the two groups $(P>0.05)$, but the number of cases who had irregular cycle status was significantly higher than that was in controls $(P=0.04)$. Alcohol consumption was ignored as all nurses marked a negative answer for this except for four persons. Three main dietary patterns came to light from factor analysis, and these were named in terms of the highest loading factor groups in each pattern (Table 3). Food groups with factor loadings over 0.20 were considered as making a significant contribution to the pattern. These three dietary patterns accounted for $29.97 \%$ of the total variance in food intake. The healthy dietary pattern was high in vegetables, fruits, natural juice, olive, tea and coffee, fish, low-fat dairy products, legumes and nuts. The Western dietary pattern was high in red and visceral meats, fast foods, vegetable oil and mayonnaise, sweets and desserts, salty snacks, refined grains, sugar and soft drinks, high-fat dairy products, spices and fried potato. The traditional dietary pattern was high in eggs, cooked potatoes, legumes and nuts, poultry, hydrogenated oil, cabbage, sweets and desserts. The OR of PMS incidence across quintiles of dietary pattern scores is shown in Table 4 . We found a significant association between the Western dietary pattern and PMS in both models. Participants in the second (OR 2.29; $95 \%$ CI 1.12, 4.66) and third (OR 3.65; $95 \%$ CI 1.75, 7.57) quintiles were more likely to experience PMS compared with those in the first quintile in the crude model. The association between PMS and the Western dietary pattern remained significant after further adjustment for age, BMI, menstrual cycle status, physical activity and energy intake (OR 2.53; $95 \%$ CI 1.18, 5.43 and OR 4.39; $95 \%$ CI 1.97, 9.81, respectively). A dose-response relationship was not evident after testing PMS across the quintiles of the Western dietary pattern. No significant relationship was found between either the healthy or traditional dietary patterns and PMS even after adjusting for potential confounders $\left(P_{\text {trend }}>0 \cdot 05\right)$.

\section{Discussion}

For the first time, we have investigated the association between dietary patterns and PMS morbidity. The results showed that the Western dietary pattern might be associated with PMS, although a distinct dose-response relationship was not found. The healthy and traditional dietary patterns did not show any significant relationship with this disorder.

Although many studies have been conducted on PMS worldwide, few researchers have focused on the relationship between food intake and PMS morbidity. In the present study, the Western dietary pattern has the greatest score for red meat and fast foods. Previous results revealed that higher consumption of red meat ${ }^{(18)}$ and fast foods and lower consumption of fruits and vegetables, fish and olive oil ${ }^{(19)}$ was related to higher depression risk. Depression is 
Table 2. Characteristics of premenstrual syndrome cases and controls

(Mean values and standard deviations; numbers and percentages)

\begin{tabular}{|c|c|c|c|c|c|}
\hline \multirow[b]{2}{*}{ Characteristics } & \multicolumn{2}{|c|}{ Cases $(n 160)$} & \multicolumn{2}{|c|}{ Controls ( $n$ 160) } & \multirow[b]{2}{*}{$P^{*}$} \\
\hline & Mean & SD & Mean & SD & \\
\hline Age (years) & 29.62 & $5 \cdot 0$ & 29.5 & $6 \cdot 3$ & 0.8 \\
\hline Serving duration (years) & $5 \cdot 3$ & $4 \cdot 3$ & $5 \cdot 4$ & $5 \cdot 6$ & 0.8 \\
\hline Bleeding period $(\mathrm{d})$ & $6 \cdot 2$ & $1 \cdot 7$ & $6 \cdot 4$ & 1.8 & 0.3 \\
\hline Weight (kg) & $61 \cdot 2$ & $10 \cdot 5$ & $61 \cdot 2$ & 9.9 & 0.9 \\
\hline Height $(\mathrm{cm})$ & 161.8 & 5.4 & $162 \cdot 4$ & $5 \cdot 7$ & 0.3 \\
\hline BMI $\left(\mathrm{kg} / \mathrm{m}^{2}\right)$ & $23 \cdot 8$ & $4 \cdot 1$ & $23 \cdot 3$ & 3.7 & 0.5 \\
\hline Waist circumference $(\mathrm{cm})$ & $78 \cdot 1$ & $9 \cdot 0$ & $77 \cdot 4$ & 8.6 & 0.5 \\
\hline Physical activity (MET/week)† & 628.5 & 998.9 & $693 \cdot 0$ & $1044 \cdot 0$ & 0.5 \\
\hline Energy intake $(\mathrm{kJ} / \mathrm{d}) \dagger$ & $11908 \cdot 1$ & 6096.9 & $11728 \cdot 2$ & $5396 \cdot 5$ & 0.3 \\
\hline Energy intake $(\mathrm{kcal} / \mathrm{d}) \dagger$ & $2846 \cdot 1$ & $1457 \cdot 2$ & $2803 \cdot 1$ & $1289 \cdot 8$ & 0.3 \\
\hline Carbohydrate intake (g/d) & 468.9 & 174.9 & 459.9 & 191.5 & 0.6 \\
\hline Protein intake $(\mathrm{g} / \mathrm{d})$ & $103 \cdot 1$ & 33.2 & $102 \cdot 2$ & 39.1 & 0.8 \\
\hline Total fat intake $(\mathrm{g} / \mathrm{d})$ & $95 \cdot 7$ & 37.9 & 94.7 & $46 \cdot 6$ & 0.8 \\
\hline Energy from carbohydrate (\%) & $62 \cdot 0$ & $7 \cdot 2$ & $61 \cdot 8$ & $6 \cdot 9$ & 0.8 \\
\hline Energy from protein (\%) & $13 \cdot 8$ & $2 \cdot 1$ & $14 \cdot 0$ & 2.4 & 0.4 \\
\hline \multirow[t]{2}{*}{ Energy from fat $(\%)$} & $28 \cdot 6$ & $5 \cdot 8$ & $28 \cdot 6$ & $5 \cdot 6$ & 0.9 \\
\hline & $n$ & $\%$ & $n$ & $\%$ & \\
\hline \multicolumn{6}{|l|}{ Menstrual cycle status } \\
\hline Regular & 124 & $77 \cdot 5$ & 138 & $86 \cdot 3$ & 0.04 \\
\hline Irregular & 36 & $22 \cdot 5$ & 22 & $13 \cdot 7$ & \\
\hline
\end{tabular}

MET, metabolic equivalent tasks.

* The $t$ test was used for parametric variables and the Mann-Whitney's $U$ test was used for non-parametric variables.

† For non-parametric variables, instead of mean and standard deviation, median and interquartile range are reported.

Table 3. Factor loading matrix for dietary pattern identified by factor analysis $(n 320)^{*}$

\begin{tabular}{lccc}
\hline Food groups & $\begin{array}{c}\text { Healthy } \\
\text { diet }\end{array}$ & $\begin{array}{c}\text { Western } \\
\text { diet }\end{array}$ & $\begin{array}{c}\text { Traditional } \\
\text { diet }\end{array}$ \\
\hline Other vegetables & 0.78 & & \\
Green leafy vegetables & 0.69 & & \\
Orange and red vegetables & 0.68 & & \\
Fruit and juice & 0.67 & & \\
Olive & 0.42 & & -0.21 \\
Pickles & 0.39 & & \\
Tea and coffee & 0.30 & & 0.28 \\
Cabbage & 0.30 & & \\
Fish & 0.29 & 0.63 & \\
Red and visceral meats and fast & & & \\
$\quad$ foods & & & \\
Vegetable oil and mayonnaise & 0.21 & 0.61 & \\
Sweets and desserts & & 0.58 & 0.27 \\
Salty snacks & & 0.56 & \\
Refined grains & & 0.56 & \\
Sugar and soft drinks & & 0.44 & \\
High-fat dairy products & & 0.43 & 0.22 \\
Spices & 0.22 & 0.38 & \\
Low-fat dairy products & 0.26 & -0.38 & 0.22 \\
Fried potato & 0.27 & 0.31 & \\
Eggs & 0.22 & & 0.65 \\
Cooked potato & & & 0.62 \\
Nuts and legumes & 0.31 & 0.31 & 0.39 \\
Poultry & & 0.30 & 0.38 \\
Hydrogenated oil & & & 0.32 \\
Percentage of variance explained & 14.0 & 10.3 & 5.7 \\
\hline Vas & & & \\
\hline
\end{tabular}

* Values $<0.20$ were excluded; Bartlett's test of sphericity $<0.0001$; Kaiser-MeyerOlkin $=0.73$; total variance $=29.9 \%$

the most outstanding feature of severe $\mathrm{PMS}^{(20)}$. Following consumption of protein or protein plus fat-rich meals, plasma concentrations of tryptophan (the essential amino acid precursor of serotonin) show a greater decrease compared with that of large neutral amino acids (LNAA). Tryptophan and LNAA compete with each other for blood-brain barrier transport ${ }^{(21)}$. Individuals with lower levels of brain serotonin are considered to be vulnerable to affective disorders such as depression ${ }^{(22)}$.

We observed that subjects with PMS consumed more refined grains and fewer vegetables and fruits. Previous studies revealed that fruit and vegetable diets with whole grains can treat mild forms of PMS and decrease the menstrual pain ${ }^{(23,24)}$. Dietary fibre intake has shown a significantly inverse relationship to menstrual pain scale ${ }^{(25)}$.

In this study, the Western dietary pattern was high in $\mathrm{Na}$ from salty meals and snacks. Studies have suggested that excess salt intake, by decreasing $\mathrm{Mg}$ levels, may worsen premenstrual symptoms ${ }^{(26)}$. In addition, Western diets with low vegetable intakes and high intakes of refined grains and meats are poor sources of $\mathrm{Mg}^{(27)}$.

Our results indicate that low-fat dairy products were inversely related to the Western dietary pattern, whereas high-fat dairy products had a positive score. It has been shown that consuming whole milk rather than skimmed or low-fat milk slightly increased the risk for $\mathrm{PMS}^{(28)}$.

Barnard et $a l .{ }^{(7)}$ investigated the effects of a low-fat, vegetarian diet on premenopausal women. Study findings showed decreased premenstrual symptoms in women, with moderate-to-intense pain in menstruation. Furthermore, a low-fat, high-fibre diet significantly decreased serum estrone sulphate levels ${ }^{(29)}$.

Vitamin D food sources such as fish and eggs did not feature in the Western dietary pattern. Previous studies found an inverse relationship between intake of fish and eggs and dysmenorrhoea $^{(23,30)}$. 
Table 4. Premenstrual syndrome across quintiles $(Q)$ of dietary pattern scores (Odds ratios and $95 \%$ confidence intervals)

\begin{tabular}{|c|c|c|c|c|c|c|c|c|c|c|c|}
\hline & \multicolumn{2}{|c|}{ Q1 } & \multicolumn{2}{|r|}{ Q2 } & \multicolumn{2}{|r|}{ Q3 } & \multicolumn{2}{|r|}{ Q4 } & \multicolumn{2}{|r|}{ Q5 } & \multirow[b]{2}{*}{$P_{\text {trend }}$} \\
\hline & OR & $95 \% \mathrm{Cl}$ & OR & $95 \% \mathrm{Cl}$ & OR & $95 \% \mathrm{Cl}$ & OR & $95 \% \mathrm{Cl}$ & OR & $95 \% \mathrm{Cl}$ & \\
\hline \multicolumn{12}{|c|}{ Healthy dietary pattern } \\
\hline $\begin{array}{l}\text { Model 1* } \\
P\end{array}$ & 1.00 & & 0.93 & $\begin{array}{l}0.47,1.87 \\
0.8\end{array}$ & 0.88 & $\begin{array}{l}0.44,1.76 \\
0.7\end{array}$ & 0.73 & $\begin{array}{l}0.36,1.46 \\
0.3\end{array}$ & $1 \cdot 20$ & $\begin{array}{l}0.60,2.42 \\
0.5\end{array}$ & $0 \cdot 8$ \\
\hline $\begin{array}{l}\text { Model 2† } \\
P\end{array}$ & 1.00 & & 0.99 & $\begin{array}{l}0.48,2.02 \\
0.9\end{array}$ & 0.99 & $\begin{array}{l}0.48,2.07 \\
0.9\end{array}$ & 0.78 & $\begin{array}{l}0.36,1.67 \\
0.5\end{array}$ & 1.54 & $\begin{array}{l}0.62,3.81 \\
0.3\end{array}$ & 0.7 \\
\hline \multicolumn{12}{|c|}{ Western dietary pattern } \\
\hline $\begin{array}{l}\text { Model } 1 \\
P\end{array}$ & 1.00 & & $2 \cdot 29$ & $\begin{array}{l}1 \cdot 12,4.66 \\
0.02\end{array}$ & $3 \cdot 65$ & $\begin{array}{l}1.75,7.57 \\
0.001\end{array}$ & $1 \cdot 22$ & $\begin{array}{l}0.59,2.49 \\
0.5\end{array}$ & 1.78 & $\begin{array}{l}0.87,3.61 \\
0.1\end{array}$ & 0.5 \\
\hline $\begin{array}{l}\text { Model } 2 \\
P\end{array}$ & 1.00 & & $2 \cdot 53$ & $\begin{array}{l}1 \cdot 18,5 \cdot 43 \\
0.01\end{array}$ & $4 \cdot 39$ & $\begin{aligned} & 1.97,9.81 \\
< & 0.001\end{aligned}$ & 1.52 & $\begin{array}{l}0.65,3.57 \\
0.3\end{array}$ & $2 \cdot 36$ & $\begin{array}{l}0.87,6.36 \\
0.08\end{array}$ & 0.2 \\
\hline \multicolumn{12}{|c|}{ Traditional dietary pattern } \\
\hline $\begin{array}{l}\text { Model } 1 \\
P\end{array}$ & 1.00 & & 0.93 & $\begin{array}{l}0.46,1.88 \\
0.8\end{array}$ & 1.00 & $\begin{array}{l}0.50,2.00 \\
1.00\end{array}$ & 0.77 & $\begin{array}{l}0.38,1.56 \\
0.4\end{array}$ & 1.89 & $\begin{array}{l}0.93,3.85 \\
0.07\end{array}$ & 0.1 \\
\hline $\begin{array}{l}\text { Model } 2 \\
P\end{array}$ & 1.00 & & 0.90 & $\begin{array}{l}0.44,1.84 \\
0.7\end{array}$ & 1.07 & $\begin{array}{l}0.52,2.19 \\
0.8\end{array}$ & 0.83 & $\begin{array}{l}0.40,1.71 \\
0.6\end{array}$ & 2.09 & $\begin{array}{l}0.92,4.74 \\
0.07\end{array}$ & 0.2 \\
\hline
\end{tabular}

${ }^{*}$ Model 1: crude.

† Model 2: adjusted for age, BMI, menstrual cycle status, physical activity and energy intake.

We observed that the Western dietary pattern included high scores for sweets, desserts, sugar and soft drinks. Results of a previous study showed that subjects with premenstrual tension consumed more refined sugar and carbohydrates than did the normal people ${ }^{(31)}$. Other studies on PMS patients showed increased intake of cakes and desserts during the late luteal phase $^{(32,33)}$. Women with severe PMS have higher cravings for high-sweet-fat foods ${ }^{(34)}$. This may be due to the effects of premenstrual low serotonin activity, which often coexists with food cravings and depression in PMS patients ${ }^{(35)}$. By eating high-glycaemic index carbohydrates, the ratio of plasma tryptophan:LNAA increases, which helps allay some of the psychological symptoms ${ }^{(36)}$.

In the present study, we observed that participants in the fourth quintile of the healthy dietary pattern had an OR of 0.73 for PMS morbidity. However, it is possible that the total intake of healthy food items by our population is insufficient to yield substantially beneficial effects.

Our findings may not be directly comparable to previous research because of differences in study design and methods and our specific population. However, in terms of foods and food groups, we arrived at the same conclusion that a Western dietary pattern with meals and snacks high in fat, sugar and salt might be related to PMS morbidity.

This study does have some limitations that may affect interpretation of our findings. First, we could not include nurses from all parts of the hospitals, because we were not allowed to enter some areas such as emergency rooms, neonatal intensivecare units and surgical, infection and cancer wards either because personnel were too busy to find time to cooperate with us or because these wards were not safe for non-medical personnel to stay in for a long time. Therefore, we missed the opportunity to recruit certain nurses who were possibly under greater work stress. Second, in trying to document food consumption among nurses over the previous year, both the quantification of portion sizes and the frequency of consumption may have been subject to recall bias. However, in spite of these biases that can occur with the use of FFQ, they are still considered the best method for gathering dietary data in large population studies - such as the Nurses' Health Study 2 cohort $^{(37)}$. Third, like all case-control studies, we could not determine the temporal relationship between dietary patterns and PMS. It is possible that premenstrual symptom experience influences diet choices (specifically preferences for fatty, salty or sweet foods) rather than the reverse. Fourth, the findings of our study might not be generalisable to other populations. One of the limitations of data-driven diet pattern analysis is that the derived patterns tend to be specific to the population under evaluation and may not easily be translated into broader recommendations for other populations with different diets and among whom correlations between foods are different. Despite these limitations, this is the first study to examine the relationship of major dietary patterns and PMS.

In conclusion, we have investigated the relationship between dietary patterns and PMS morbidity in a nurse population that has high work pressures and responsibilities. Among this population, we found that a Western dietary pattern might be associated with increased PMS morbidity. However, this result should be interpreted with caution because of the lack of a dose-response relationship. Further studies are needed to confirm this finding.

\section{Acknowledgements}

The authors would like to thank the hospital staff and all nurses for their participation, cooperation and collaboration in this study.

This research has been supported by Tehran University of Medical Sciences and Health Services grant no. 24367-161-03-92.

The authors' contributions are as follows: F. S. and G. S. contributed to the study design and revised the manuscript. N. F. and K. A. contributed to data collection and wrote the manuscript. M. Q. and F. K. contributed to data analysis and 
interpretation of the data. All authors listed approved the content of the submitted manuscript.

There are no conflicts of interests to declare.

\section{References}

1. American College of Obstetricians and Gynecologists Practice Bulletin (2000) Clinical management guidelines for obstetriciangynecologists. Am J Obstet Gynecol 95, 1-9.

2. Steiner M \& Born L (2000) Diagnosis and treatment of premenstrual dysphoric disorder: an update. Int Clin Psychopharmacol 15, Suppl. 3, S5-S17.

3. Schmidt PJ, Nieman LK, Danaceau MA, et al. (1998) Differential behavioral effects of gonadal steroids in women with and in those without premenstrual syndrome. $N$ Engl J Med 338, 209-216.

4. Head K (1997) Premenstrual syndrome: nutritional and alternative approaches. Altern Med Rev 2, 12-25.

5. Kendler KS, Karkowski LM, Corey LA, et al. (1998) Longitudinal population-based twin study of retrospectively reported premenstrual symptoms and lifetime major depression. Am J Psychiatry 155, 1234-1240.

6. Gorbach SL \& Goldin BR (1987) Diet and the excretion and enterohepatic cycling of estrogens. Prev Med 16, 525-531.

7. Barnard ND, Scialli AR, Hurlock D, et al. (2000) Diet and sexhormone binding globulin, dysmenorrhea, and premenstrual symptoms. Obstet Gynecol 95, 245-250.

8. Abraham GE \& Rumley RE (1987) Role of nutrition in managing the premenstrual tension syndromes. J Reprod Med 32, 405-422.

9. Cheng SH, Shih CC, Yang YK, et al. (2013) Factors associated with premenstrual syndrome - a survey of new female university students. Kaobsiung J Med Sci 29, 100-105.

10. Seedhom AE, Mohammed ES \& Mahfouz EM (2013) Life style factors associated with premenstrual syndrome among El-Minia University Students, Egypt. Int Sch Res Notices $\mathbf{2 0 1 3}$ Article ID 617123.

11. Namavar Jahromi B, Pakmehr S \& Hagh-Shenas H (2011) Work stress, premenstrual syndrome and dysphoric disorder: are there any associations? Iran Red Crescent Med J 13, 199-202.

12. Park YH, Jeong BS, Kim CY, et al. (2001) Prevalence of premenstrual dysphoric disorder and occupational function in a nurse group. J Korean Neuropsychiatr Assoc 40, 832-841.

13. Steiner M, Macdougall M \& Brown E (2005) The premenstrual symptoms screening tool (PSST) for clinicians. Arch Womens Ment Health 6, 203-209.

14. Hariri FZ, Moghaddam-Banaem L, Siah Bazi S, et al. (2013) The Iranian version of the Premenstrual Symptoms Screening Tool (PSST): a validation study. Arch Womens Ment Health 16, 531-537.

15. National Institutes of Health (2015) What are menstrual irregularities? https://www.nichd.nih.gov/health/topics/menstruation/ conditioninfo/Pages/irregularities.aspx (accessed April 2015).

16. Esfahani FH, Asghari G, Mirmiran P, et al. (2010) Reproducibility and relative validity of food group intake in a food frequency questionnaire developed for the Tehran Lipid and Glucose Study. J Epidemiol 20, 150-158.

17. Moghaddam MHB, Aghdam FB, Jafarabadi MA, et al. (2012) The Iranian version of international physical activity questionnaire (IPAQ) in Iran: content and construct validity, factor structure, internal consistency and stability. World Appl Sci J 18, 1073-1080.
18. Sánchez-Villegas A, Delgado-Rodríguez M, Alonso A, et al. (2009) Association of the Mediterranean dietary pattern with the incidence of depression: the Seguimiento Universidad de Navarra/University of Navarra follow-up (SUN) cohort. Arch Gen Psychiatry 66, 1090-1098.

19. Sanchez-Villegas A, Toledo E, de Irala J, et al. (2012) Fast-food and commercial baked goods consumption and the risk of depression. Public Health Nutr 15, 424-432.

20. Ko CH, Long CY, Chen SY, et al. (2013) Depression, irritability, and anxiety in women with premenstrual dysphoric disorder. Int J Psychiatry Med 46, 39-55.

21. Wurtman RJ, Wurtman JJ, Regan MM, et al. (2003) Effects of normal meals rich in carbohydrates or proteins on plasma tryptophan and tyrosine ratios. Am J Clin Nutr 77, 128-132.

22. Markus CR, Firk C, Gerhardt C, et al. (2008) Effect of different tryptophan sources on amino acids availability to the brain and mood in healthy volunteers. Psychopharmacology (Berl) 201, 107-114.

23. Balbi C, Musone R, Menditto A, et al. (2000) Influence of menstrual factors and dietary habits on menstrual pain in adolescence age. Eur J Obstet Gynecol Reprod Biol 91, 143-148.

24. Tekač I \& Kotnik B (2009) Premenstrual syndrome. Zdrav Vestn 78, 555-563.

25. Nagata C, Hirokawa K, Shimizu N, et al. (2005) Associations of menstrual pain with intakes of soy, fat and dietary fiber in Japanese women. Eur J Clin Nutr 59, 88-92.

26. Johnson S (2001) The multifaceted and widespread pathology of magnesium deficiency. Med Hypotheses 56, 163-170.

27. Beyer PL (2012) Intake: digestion, absorption, transport, and excretion of nutrients. In Krause's Food \& the Nutrition Care Process, 13th ed. pp. 2-250 [LK Mahan, S Escott-Stump and JL Raymond, editors]. St. Louis, MO: Elsevier Saunders.

28. Bertone-Johnson ER, Hankinson SE, Bendich A, et al. (2005) Calcium and vitamin D intake and risk of incident premenstrual syndrome. Arch Intern Med 165, 1246-1252.

29. Woods MN, Gorbach SL, Longcope C, et al. (1989) Low-fat, high-fiber diet and serum estrone sulfate in premenopausal women. Am J Clin Nutr 49, 1179-1183.

30. Di Cintio E, Parazzini F, Tozzi L, et al. (1997) Dietary habits, reproductive and menstrual factors and risk of dysmenorrhoea. Eur J Epidemiol 13, 925-930.

31. Goei GS, Ralston JL \& Abraham GE (1982) Dietary patterns of patients with premenstrual tension. J Appl Nutr 34, 4-11.

32. Wurtman JJ, Brzezinski A, Wurtman RJ, et al. (1989) Effect of nutrient intake on premenstrual depression. Am J Obstet Gynecol 161, 1228-1234.

33. Cross GB, Marley J, Miles H, et al. (2001) Changes in nutrient intake during the menstrual cycle of overweight women with premenstrual syndrome. Br J Nutr 85, 475-482.

34. Yen JY, Chang SJ, Ko CH, et al. (2010) The high-sweet-fat food craving among women with premenstrual dysphoric disorder: emotional response, implicit attitude and rewards sensitivity. Psychoneuroendocrinology 35, 1203-1212.

35. Dye L \& Blundell JE (1997) Menstrual cycle and appetite control: implications for weight regulation. Hum Reprod 12, $1142-1151$

36. Murakami K, Sasaki S, Takahashi Y, et al. (2008) Dietary glycemic index is associated with decreased premenstrual symptoms in young Japanese women. Nutrition 24, 554-561.

37. Colditz GA \& Hankinson SE (2005) The Nurses' Health Study: lifestyle and health among women. Nat Rev Cancer $\mathbf{5}$, 388-396. 